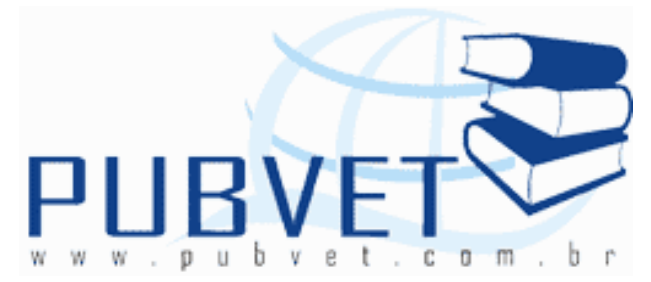

PUBVET, Publicações em Medicina Veterinária e Zootecnia.

\title{
Avaliação do ganho em peso de cordeiros da raça Santa Inês no periparto criados na região do Planalto Central brasileiro
}
Adalto José de Souza ${ }^{1}$; Rodrigo Zaiden Taveira ${ }^{2}$; Osvaldo José da Silveira Neto $^{2}$; Leonardo Capelle Melo ${ }^{3}$

${ }^{1}$ Docente - IFTGO - Ceres, GO

${ }^{2}$ Docente e Pesquisador - Curso de Zootecnia - UEG - UnU - São Luís de Montes Belos, GO

${ }^{3}$ Acadêmico do curso de Zootecnia - UEG - UnU - São Luís de Montes Belos, GO

\section{Resumo}

A atividade de ovinocultura de corte tem evoluído e expandido nos últimos anos, atraindo inúmeros empresários a investirem no potencial da atividade. Sendo assim, o objetivo desse estudo consistiu em avaliar o ganho em peso de cordeiros da raça Santa Inês, no periparto, criados na região do Planalto Central. Os dados utilizados foram provenientes de três rebanhos distintos (rebanho 01; rebanho 02 e rebanho 03). No rebanho 01, foiavaliado o peso e os ganhos em peso até os 60 dias de idade em sistema à pasto comparado ao sistema de confinamento pós-parto. No rebanho 02foram consideradas duas estações do ano (secas e águas), avaliando seus efeitosno tipo de nascimento (simples e gemelar) e ganho em peso. No rebanho 03 foi avaliada a influência das duas estações do ano (secas e águas) sobreos machos e fêmeas oriundos 
SOUZA, A.J. et al. Avaliação do ganho em peso de cordeiros da raça Santa Inês no periparto criados na região do Planalto Central brasileiro. PUBVET, Londrina, V. 8, N. 7, Ed. 256, Art. 1698, Abril, 2014.

de partos simples. As análises estatísticas foram realizadas mediante a utilização do programa estatístico SAS e as médias das características comparadas por meio do teste $t(p<0,05)$. Os resultados do rebanho 01indicaram haver superioridade no desempenho dos animaismantidos no sistema de confinamento no pós-parto. No rebanho 02 houve superioridade no período de águas e para cordeiros oriundos de partos simples. No rebanho 03 ocorreu desempenho semelhante para os períodos de águas e secas com superioridade para machos. O confinamento total dos cordeiros com suplementação promoveu bons resultados em ambas asestação avaliadas.

Palavras-chave: desempenho; ovinos; sazonalidade.

\title{
Weight gain evaluation in Santa Inês lambs in the periparturition proceeding of Brazilian Central tableland
}

\begin{abstract}
The beef goat activity has been developed and expanded in the last years, attracting lots of business men to invest in the activity potentials. The aim of this study was to evaluate the body weight gain in lambs of Santa Inês breed, in the periparturition, proceeding from Brazilian Central tableland. The data used were provided from three different livestock (livestock 01; livestock 02 and livestock 03). In the livestock 01 was to evaluated the weight and weight gains until the 60 days of age in pasture system compared topostbirthfeedlot system. In the livestock 02 was considered two year seasons (dry and water station) evaluating theirs effects upon the kind of birth (simple and twin) andweight gain. In the livestock 03 was evaluated the influence of two year season (dry and water station) upon the male and female of simple birth. The statistical analyses were carried out by the SAS statistical program and the means were compared by the test $(p<0.05)$. The results from livestock 01 showed superiority in the performance of animals kept in the feedlot system in the postpartum. In the livestock 02 was superiority considering the water station and for lambs from simple parturition. In the livestock 03 occurred
\end{abstract}


SOUZA, A.J. et al. Avaliação do ganho em peso de cordeiros da raça Santa Inês no periparto criados na região do Planalto Central brasileiro. PUBVET, Londrina, V. 8, N. 7, Ed. 256, Art. 1698, Abril, 2014.

similar performancefor the water and dry station, with superiority for the males. The handling of sheep feedlot handling before the partition and total feedlot of lambs with supplementation. The total lams feedlot with supplementation promoted good results in both evaluated season.

Keywords: ovine;performance; seasonality.

\section{INTRODUÇÃO}

A otimização do crescimento pré e pós-natal de cordeiros nos sistemas de produção por meio de manejos adequados do ponto de vista alimentar e sanitário, naturalmente acompanhados pelas escriturações zootécnicas e boa interpretação dos dados nela contida, promoves profissionalização da atividade proporcionando benefícios econômicos (Geraseev et al., 2006).

As interpretações das curvas de crescimento de cordeiros na fase de lactentes e desmamados acompanhados pelos estudos dos fatores ambientais e genéticos podem trazer informações valiosas na tomada de decisão dentro dos criatórios (Sarmento et al., 2006).

A composição corporal e o ganho em peso em ovinos estão intimamente correlacionados, sendo que alguns fatores como o estado de desenvolvimento animal, raça, sexo, tipo de nascimento, época e ano de nascimento, nutrição e sanidade influenciam, em parte, a composição corporal e o ganho em peso (Pires et al., 2000).

$\mathrm{Na}$ fase de crescimento do nascimento até a puberdade, entre o quinto e o sexto meses de vida para a raça Santa Inês, os cordeiros apresentam máxima capacidade de ganho em peso, apresentando alta conversão alimentar. Sobretudo, na fase de lactentes ocorre o maior ganho, relacionado com a maior exigência nutricional dos animais (Bernardi et al., 2005; Alves et al., 2006).

Pérez et al. (2005), acrescentam ainda que o crescimento pós-natal é linear durante as 10 primeiras semanas de vida, originando posteriormente, uma curva decrescente com tendência horizontal, proporcional a curva de 
SOUZA, A.J. et al. Avaliação do ganho em peso de cordeiros da raça Santa Inês no periparto criados na região do Planalto Central brasileiro. PUBVET, Londrina, V. 8, N. 7, Ed. 256, Art. 1698, Abril, 2014.

lactação que apresenta pico entre a $3^{a}$ e $4^{a}$ semana de lactação e $80 \%$ de sua produção por volta da $8^{a}$ e $10^{a}$ semana após o parto.

Com o declínio da produção láctea da ovelha e aumento da exigência dos cordeiros, se faz necessário alimentação suplementar com alimento de boa qualidade a fim de suprir as necessidades dos cordeiros, visado desmamá-los com pesos expressivos. Este procedimento é importante e deve ser incorporado aos sistemas de produção, pois traz benefícios nos índices de sobrevivência dos cordeiros e evidentemente nos ganhos finais quando os animais vão ao abate, ou mesmo para reposição de rebanho (Bueno et al., 2007).

No aspecto sanitário, ocorre redução temporária da imunidade das ovelhas no periparto e consequente elevação dos níveis de infecção por parasitas, resultando aumento na eliminação de ovos de parasitos nas fezes de matrizes, provocando elevação na contaminação de pastagens por lavas, aumentando a infecção das próprias ovelhas. Em relação aos animais jovens, sabe-se que há maior susceptibilidade e infecção dos parasitos e que idades mais avançadas sob infecção gradual possibilitam aos animais o estabelecimento de níveis crescentes de imunidade adquirida (Cunha et al., 2004).

A literatura evidencia bons resultados com sistema que utilizam o confinamento de matrizes no periparto, e confinamento totais de crias até o abate e/ou seleção com finalidade de minimizar essa infestação das pastagens e infecção de matrizes e crias resultando em melhores desempenhos reprodutivos e produtivos (Siqueira et al., 1993; Cunha et al., 2004; Cunha et al., 2007).

Face ao exposto, esse estudo teve como objetivo avaliar o desempenho produtivo de cordeiros da raça Santa Inês no periparto, criados na região no Planalto Central, provenientes de três distintos rebanhos. 
SOUZA, A.J. et al. Avaliação do ganho em peso de cordeiros da raça Santa Inês no periparto criados na região do Planalto Central brasileiro. PUBVET, Londrina, V. 8, N. 7, Ed. 256, Art. 1698, Abril, 2014.

\section{MATERIAL E MÉTODOS}

Os dados utilizados neste trabalho foram provenientes de três rebanhos distintos, denominadas: rebanho 01; rebanho 02 e rebanho 03, localizados nos municípios de Brasília-DF, Unaí-MG e Águas Lindas-GO, respectivamente.

O rebanho 01 adotava o sistema de confinamento de mães e crias após o parto até o desmame. Nesse período os cordeiros tinham acesso à ração no creep-feeding, sendo que após o desmame os cordeiros permaneciam confinado e as matrizes voltavam ao pasto para um novo ciclo reprodutivo. Nesse rebanho as características avaliadas foram: peso ao nascer (PN); peso aos 60 dias de vida (P60); com manejo à pasto (Abril e Maio) com manejo confinado (Setembro).

O rebanho 02 utilizava o sistema intensivo de criação, com confinamento total de mães e crias, no qual os cordeiros permaneciam integralmente com as mães até os 30 dias iniciais de lactação na maternidade, com acesso ao creepfeeding.A partir dos 30 dias recebiam alimentação ad libitum e mamadas controladas duas vezes ao dia até o desmame.Nesse rebanho foram avaliados o PN e P60 em duas estações do ano bem definidas (estação seca: Junho, Julho e Agosto) e (estação de águas: Janeiro, Fevereiro e Março). Foram avaliados ainda os ganhos em peso de cordeiros lactentes nos três primeiros meses de idade,tendo em vista o tipo de nascimento (simples e duplo), observando sua influência no PN; P30; P60; GMD1 (0-30dias); GMD2 (3060dias) e GMD3 (0-60dias).

O rebanho 03 utilizava o sistema semi-intensivo de criação de matrizes, adicionando o sistema intensivo de confinamento no periparto das matrizes e confinamento total de cordeiros. Neste sistema, mães e crias permaneciam juntas até 30 dias após o parto em uma maternidade. Posteriormente a esta fase os cordeiros eram conduzidos a uma baía para lactentes e as ovelhas para o pasto durante o dia, retornando ao aprisco no final da tarde. As mamadas controladas ocorriam no período da manhã e no final da tarde até o desmame. 
SOUZA, A.J. et al. Avaliação do ganho em peso de cordeiros da raça Santa Inês no periparto criados na região do Planalto Central brasileiro. PUBVET, Londrina, V. 8, N. 7, Ed. 256, Art. 1698, Abril, 2014.

Nesse rebanho foram avaliados o PN e P60 para machos e fêmeas provenientes de partos simples.

As análises estatísticas foram realizadas mediante a utilização do programa estatístico SAS e as médias das características comparadas por meio do teste $t(p<0,05)$.

\section{RESULTADOS E DISCUSSÃO}

\section{Rebanho 01}

Avaliando o peso ao nascer (PN) dos dois sistemas de criação, verificouse que não houve diferença estatística significativa $(p<0,05)$ quanto ao sistema de criação, tendo em vista que a mudança do manejo ocorreu após o nascimento e as condições de pré-parto ainda ocorriam a pasto. Em relação ao (P60) nota-se diferença estatística significativa $(p<0,05)$, evidenciada pela superioridade no sistema com confinamento durante a fase de lactação.

A tabela 1 apresenta dados referentes aos valores médios de peso ao nascer (PN), peso aos 60 dias (P60) e ganhos médios diários (GMD) de machos (M) e fêmeas (F) em dois sistemas de criação no período seco do ano.

As médias para peso ao nascer encontradas nesse estudo estão próximas aos 3,68 kg e 3,85 kg, registrados pro Freitas et al. (2005) para animais da raça Santa Inês criados em sistema de campo nativo, suplementados apenas na seca criados em brachiariahumidícula. Esses resultados foram ainda superiores ao $3,39 \mathrm{~kg}$ registrado por Girão et al. (1999) no estado do Piauí e por Silva \& Araújo (2000) os quais encontraram valores variando entre 2,84 a $3,54 \mathrm{Kg}$ em 08 fazendas no estado do Ceará.

Tendo em vista o peso e ganho médios até os 60 dias, Freitas et al. (2005) avaliando cordeiros em pastagem e com acesso ao alimento privativo das 17:00 às 7:00 (creep-feeding-noturno), encontraram (P60) de 13,53 kg, com GMD de 0,161. Esses resultados foram muito semelhantes aos encontrados por Siqueira et al. (1993) os quais observaram ganhos para 
SOUZA, A.J. et al. Avaliação do ganho em peso de cordeiros da raça Santa Inês no periparto criados na região do Planalto Central brasileiro. PUBVET, Londrina, V. 8, N. 7, Ed. 256, Art. 1698, Abril, 2014.

animais aos 60 dias de 0,153 kg/dia em confinamento e de 0,082 kg/dia em animais mantido a pasto. Sobre esses baixos ganhos a pasto Silva \& Araújo (2000) encontraram variação de 0,066 a 0,131 kg/dia até o $84^{\circ}$ dia de idade.

Tabela 1. Média e desvio-padrão do peso ao nascer (PN) e peso aos 60 dias de idade (P60) e ganhos médios diários (GMD) de machos (M) e fêmeas $(F)$ em sistemas de criação à pasto e confinado no período de seca em rebanho localizado na região do Planalto Central Brasileiro.
PN
$\mathrm{P} 60(\mathrm{Kg})$
GMD (Kg)

Sexo Sistema Média \pm DP N Média \pm DP N Média \pm DP

$\begin{array}{llllll}\text { Pasto } & 4,06 \pm 0,4^{\text {ns }} & 24 & 7,76 \pm 1,63^{*} & 24 & 0,062 \pm 0,009\end{array}$

M Confinado $3,83 \pm 0,74^{\text {ns }} 12 \quad 13,92 \pm 3,02^{*} \quad 12 \quad 0,176 \pm 0,001$

Pasto $\quad 3,63 \pm 0,57^{\text {ns }} \quad 32 \quad 7,12 \pm 1,77^{*} \quad 32 \quad 0,058 \pm 0,017$

F Confinado $3,73 \pm 0,50^{\text {ns }} \quad 16 \quad 13,33 \pm 3,35^{*} \quad 16 \quad 0,160 \pm 0,020$

* significativo pelo teste $t(p<0,05)$

${ }^{n s}$ não significativo pelo teste $t(p>0,05)$

No caso do sistema a pasto no qual estava inserido o rebanho 01 , pode ter ocorrido soma de diversos fatores limitantes, tais como problemas alimentar e sanitário, refletindo no desempenho dos cordeiros, tanto pela baixa ingestão láctea quanto por altas taxas de infecções. Acrescenta-se ainda que devido à busca dos cordeiros por alimentos sólidos ser inversamente proporcional a produção de leite da ovelha, e o alimento sólido disponível seria a pastagem, a qual poderia estar contaminada, ou com baixa qualidade nutricional.

Bernardi et al. (2005), relataram que a criação em pastejo pode ser variável, sendo a verminose o fator limitante, provocando redução no consumo 
SOUZA, A.J. et al. Avaliação do ganho em peso de cordeiros da raça Santa Inês no periparto criados na região do Planalto Central brasileiro. PUBVET, Londrina, V. 8, N. 7, Ed. 256, Art. 1698, Abril, 2014.

voluntario de alimentos e prejuízos na digestão e absorção de nutrientes, ocasionando redução no ganho em peso dos animais.

No entanto, pelos valores apresentados na tabela 1, nota-se que já houve melhoria nos ganhos com a implantação do confinamento pós-parto, podendo até realizar o desmame aos 60 dias, entretanto, encontram-se abaixo do esperado para os dois sistemas.

Em relação aos sistemas é possível obter melhores ganhos desde que as ovelhas tenham condições nutricionais e sanitárias para produzir leite suficiente para alimentar sua cria e, que além do leito o cordeiro tenha acesso a alimento de boa qualidade. De acordo com Borges (2000), essas condições intensificam-se no terço final de gestação, onde ocorre a $1^{\text {a }}$ fase de lactação, sendo necessário a ovelha acumular gordura para ser mobilizada no início de lactação em que a ovelha se encontra em déficit energético.

\section{Rebanho 02}

Pode ser observado na tabela 2 que houve diferença estatística significativa $(p<0,05)$ entre as estações avaliadas, sendo evidenciado superioridade no período de incidência de chuvas, o que seria normal para animais criados à pasto devido a sazonalidade de oferta e qualidades das forrageiras, entretanto, o sistema de manejo utilizado nesta propriedade é confinado, buscando potencializar o crescimento durante o ano todo.

A tabela 2 apresenta os valores médios de peso ao nascer (PN) e peso corrigido para os 60 dias (P60) e ganhos médios diários (GMD) de machos (M) e fêmeas $(F)$ em duas estações do ano.

Geraseev et al. (2006) avaliando efeitos das restrições alimentares pré e pós-natal sobre o crescimento e o desempenho de cordeiros Santa Inês do nascimento ao desmame encontrou $\mathrm{PN}(\mathrm{kg})$ e $\mathrm{GMD}(\mathrm{kg} / \mathrm{dia})$ para os grupos sem restrições, com restrições pós e pré-natal de 4,60,17,12 e 0,200; 4,40,14,15 e 0,$153 ; 3,00,13,00$ e $0,162 \mathrm{Kg}$, respectivamente. Valores estes semelhantes 
SOUZA, A.J. et al. Avaliação do ganho em peso de cordeiros da raça Santa Inês no periparto criados na região do Planalto Central brasileiro. PUBVET, Londrina, V. 8, N. 7, Ed. 256, Art. 1698, Abril, 2014.

em situações e resultados encontrados no rebanho desse estudo(rebanho 02), concluindo que cordeiros na estação de secas sofreram essas restrições e mesmo com alimentação ad libitum na fase de aleitamento não atingem a mesma taxa de crescimento e o mesmo peso à desmama dos animais que não sofreram restrição, como reflexo do seu baixo peso ao nascer, que afeta o consumo de alimento líquido ao longo desta fase.

Tabela 2. Média e desvio-padrão do peso ao nascer (PN) e peso aos 60 dias de idade (P60) e ganhos médios diários (GMD) de machos (M) e fêmeas (F) nas estações da seca e das águas, em rebanho localizado na região do Planalto Central Brasileiro.

Estação das águas Estação das águas

Sexo Idade $\mathrm{N} \quad$ Média $\pm D P \quad \mathrm{GMD}(\mathrm{Kg}) \quad \mathrm{N}$ Média $\pm D P \quad \mathrm{GMD}(\mathrm{Kg})$

$\begin{array}{lllll}P N(K g) & 28 & 3,30 \pm 0,58^{*} & 30 & 4,36 \pm 0,91^{*}\end{array}$

$\begin{array}{llllllll}\text { M } & \text { P60 (Kg) } & 28 & 13,22 \pm 2,72^{*} & 0,165 & 30 & 18,69 \pm 3,80^{*} & 0,239 \\ & \text { PN (Kg) } & 16 & 3,46 \pm 0,53^{*} & & 28 & 3,97 \pm 0,61^{*} & \\ & & & & & & & \\ \text { F } & \text { P60 (Kg) } & 16 & 13,46 \pm 2,02^{*} & 0,167 & 28 & 16,93 \pm 2,95^{*} & 0,216\end{array}$

* significativo pelo teste $t(p<0,05)$

ns não significativo pelo teste $t(p>0,05)$

Diante deste contexto, nesta situação pode ter ocorrido alguma falha nutricional no período seco do ano afetando a fase pré e pós-natal, pois os pesos e ganhos no período das águas foram elevados para machos e fêmeas desta raça, mostrando que a raça tem potencial para produção de cordeiros com pesos expressivos. No entanto, com melhorias no planejamento alimentar para a entressafra pode evitar estas sequelas, refletindo em maiores ganhos em peso dos cordeiros. 
SOUZA, A.J. et al. Avaliação do ganho em peso de cordeiros da raça Santa Inês no periparto criados na região do Planalto Central brasileiro. PUBVET, Londrina, V. 8, N. 7, Ed. 256, Art. 1698, Abril, 2014.

Verificou-se também diferença estatística significativa $(p<0,05)$ tendo em vista o tipo de nascimento, tendo havido superioridade para os cordeiros oriundos de partos simples. $O$ fato de as crias de partos simples apresentarem maior peso ao nascer e melhor desempenho que as de parto gemelar pode, em parte, ser explicado pelo maior aporte nutricional, ainda na gestação, que é oferecido para o feto (PN) e pela inexistência de competição nutricional entre crias na amamentação.

A tabela 3 apresenta os dados referentes aos valores médios de (PN), (P30) e (P60) e GMD(0-30), GMD(30-60) e GMD(0-60) para machos (M) e fêmeas $(F)$ oriundos de partos simples e duplos.

Tabela 3. Média e desvio-padrão do peso ao nascer (PN); peso aos 30 dias de idade (P30); peso aos 60 dias de idade (P60) e ganhos médios diários(GMD) do nascimento até os 60 dias de idade para machos (M) e fêmeas (F) nascidos de parto simples e duplo em rebanho localizado na região do Planalto Central Brasileiro.

\begin{tabular}{|c|c|c|c|c|c|c|c|}
\hline \multirow[t]{3}{*}{ Idade } & \multirow[t]{3}{*}{ Nascimento } & \multicolumn{3}{|c|}{ Machos } & \multicolumn{3}{|c|}{ Fêmeas } \\
\hline & & $\mathrm{N}$ & Média $\pm D P$ & GMD & $\mathrm{N}$ & Média $\pm D P$ & GMD \\
\hline & & & & (0-60 dias) & & & (0-60 dias) \\
\hline \multirow[t]{2}{*}{$\mathrm{PN}(\mathrm{Kg})$} & Simples & 30 & $4,36 \pm 0,91^{*}$ & & 28 & $3,97 \pm 0,61^{\mathrm{ns}}$ & \\
\hline & Duplo & 16 & $3,05 \pm 0,79^{*}$ & & 10 & $3,52 \pm 0,41^{\text {ns }}$ & \\
\hline \multirow[t]{2}{*}{ P30 (Kg) } & Simples & 30 & $11,40 \pm 2,61^{*}$ & & 28 & $10,13 \pm 1,38^{*}$ & \\
\hline & Duplo & 16 & $7,16 \pm 1,21^{*}$ & & 10 & $7,27 \pm 1,27 *$ & \\
\hline \multirow[t]{2}{*}{ P60 (kg) } & Simples & 30 & $18,69 \pm 3,80^{*}$ & 0,239 & 28 & $16,35 \pm 3,26 *$ & 0,206 \\
\hline & Duplo & 16 & $13,51 \pm 2,00^{*}$ & 0,174 & 10 & $13,22 \pm 1,42 *$ & 0,162 \\
\hline
\end{tabular}

* significativo pelo teste $t(p<0,05)$

ns não significativo pelo teste $t(p>0,05)$

Pode ser destacado que os ganhos médios diários do nascimento até os 30 dias de animais provenientes de parto gemelar foram muito inferiores aos ganhos dos animais provenientes de parto simples, aproximadamente 100 g/dia para ambos os sexos. Frescura et al., 2005 ressaltam que a produção de 
SOUZA, A.J. et al. Avaliação do ganho em peso de cordeiros da raça Santa Inês no periparto criados na região do Planalto Central brasileiro. PUBVET, Londrina, V. 8, N. 7, Ed. 256, Art. 1698, Abril, 2014.

leite não é proporcional ao número de cordeiros e que nessa idade os cordeiros têm pouca habilidade em digerir alimentos sólidos, mas que são importantes para estimular 0 contato com alimentos sólidos 0 que promove 0 desenvolvimento ruminal.

Ressalta-se ainda que o ganho médio diário do $P(30)$ ao $P(60)$ foi superior para os animais de partos simples, essa diferença é menos expressiva mostrando a importância da suplementação tanto para animais de partos simples como de parto duplo. Segundo Bueno et al. (2007) até os 30 dias o cordeiro é nutrido principalmente pelo leite materno e aos poucos vão suprindo suas exigências com alimentação solida, devido a redução da produção láctea e aumento do aporte de nutrientes exigido pelo cordeiro para expressar seu potencial de crescimento.

Os dados encontrados no rebanho 02 encontram-se muito próximos aos valores observados por Costa et al. (2003) em rebanho do Instituto de Zootecnia (IZ). Em ambos os casos, percebe-se a preocupação com o conhecimento fisiológico dos animais, notando a importância da suplementação no final de gestação, o confinamento no periparto e lactação e a importância do arraçoamento e da mamada controlada, pois esses fatores somados permitem três partos a cada dois anos, alta sobrevivência de cordeiros ao desmame com pesos expressivos e principalmente um manejo que permite maior controle parasitário.

\section{Rebanho 03}

Pode ser observado, pela análise da tabela 03, que não houve diferença estatística significativa ( $p>0,05)$ entre as estações (secas e águas), atingindo bons resultados e desmamando precocemente os cordeiros com pesos expressivos, semelhantes aos dados de Geraseev et al. (2006) e aos períodos de chuvas avaliados no rebanho 02. De acordo com Siqueira (1993), ganhos acima de $0,200 \mathrm{~kg} /$ dia são considerados bons para produção de cordeiros. A 
SOUZA, A.J. et al. Avaliação do ganho em peso de cordeiros da raça Santa Inês no periparto criados na região do Planalto Central brasileiro. PUBVET, Londrina, V. 8, N. 7, Ed. 256, Art. 1698, Abril, 2014.

raça Santa Inês apresenta essa potencialidade quando tem condições favoráveis a expressarem seu potencial, todavia é uma raça em processo de formação e seleção para melhorar seu ponderal e qualidade na carcaça.

Frescura et al. (2005) observaram em mestiços Ile de France x Texel, criados em pastagem cultivada de Azevém, com creep-feeding disponível aos cordeiros, GMD de 0,332 kg/dia. Tonetto et al.(2004), por sua vez, obtiveram GMD de 0,404; 0,325 e 0,213 em pastagem cultivada de Azevém, pastagem natural suplementa e confinamento total, respectivamente.

Garcia (2002) estudando níveis de energia na ração de creep-feeding para cordeiros verificou que os cordeiros mestiços de Suffolk ganharam 0,403 $\mathrm{kg} / \mathrm{dia}$ e atingindo abate aos 61 dias. Neste estudo as ovelhas foram suplementadas com feno no período noturno ração concentrada na quantidade de $1 \%$ do Peso Vivo, ressaltando que as ovelhas lactantes necessitam de grandes quantidades de MS e que alimentação seca de boa qualidade aumenta e ingestão, tendo em vista que alimento muito úmido limita a capacidade de ingestão por capacidade de enchimento ruminal.

Tabela 4. Média e desvio-padrão do peso ao nascer (PN) e peso aos 60 dias (P60) e ganhos médios diários (GMD) de machos (M) e fêmeas (F) oriundos de partos simples em duas estações do ano em rebanho localizado na região do Planalto Central Brasileiro.

\begin{tabular}{|c|c|c|c|c|c|c|c|}
\hline \multirow[b]{2}{*}{ Sexo } & \multirow[b]{2}{*}{ Idade } & \multicolumn{3}{|c|}{ Estação de seca } & \multicolumn{3}{|c|}{ Estação de água } \\
\hline & & $\mathrm{N}$ & Média $\pm D P$ & GMD $(\mathrm{Kg})$ & $\mathrm{N}$ & Média $\pm D P$ & GMD $(\mathrm{Kg})$ \\
\hline \multirow[t]{2}{*}{$M$} & PN (Kg) & 18 & $4,24 \pm 0,84$ & 0,218 & 18 & $3,93 \pm 0,57^{\mathrm{ns}}$ & 0,214 \\
\hline & P60 (Kg) & 18 & $17,32 \pm 2,42$ & & 18 & $16,80 \pm 2,49^{\mathrm{ns}}$ & \\
\hline \multirow[t]{2}{*}{$\mathrm{F}$} & PN (Kg) & 16 & $3,59 \pm 0,82$ & 0,183 & 14 & $3,70 \pm 0,71^{\mathrm{ns}}$ & 0,173 \\
\hline & P60 (Kg) & 16 & $14,51 \pm 2,16$ & & 14 & $14,06 \pm 1,58^{\mathrm{ns}}$ & \\
\hline
\end{tabular}

* significativo pelo teste $t(p<0,05)$

${ }^{\text {ns }}$ não significativo pelo teste $t(p>0,05)$ 
SOUZA, A.J. et al. Avaliação do ganho em peso de cordeiros da raça Santa Inês no periparto criados na região do Planalto Central brasileiro. PUBVET, Londrina, V. 8, N. 7, Ed. 256, Art. 1698, Abril, 2014.

\section{CONCLUSÃO}

Tendo e vista o rebanho 01 pode ser percebido que a verminose das pastagens for o fator limitante do ganho em peso, sugere-se, portanto, o confinamento das matrizes, no mínimo após o parto, melhorando o desempenho dos cordeiros.

No rebanho 02 o decréscimo no desempenho dos animais na entre safra forrageira pode ser evitado mediante o confinamento total, desde que tenha bom planejamento alimentar, respeitando e atendendo as exigências nutricionais dos animais. A suplementação de cordeiros com alimentos sólidos de boa qualidade faz-se necessária aos cordeiros que estão em desenvolvimento da sua capacidade ruminal.

No rebanho 03 o manejo de confinamento de ovelhas no periparto e confinamento total dos cordeiros com suplementação promoveu bons resultados na estação de secas e de águas.

\section{REFERÊNCIAS BIBLIOGRÁFICAS}

BERNARDI, José Reinaldo de Amorim et al. Desempenho de Cordeiros sob Quatro Sistema de Produção. Revista Brasileira Zootecnia, v.34, n.4, p.1248-1255, 2005.

BORGES, Iran. Manejo da ovelha gestante e sua importância na criação do cordeiro. In: ENCONTRO MINEIRO DE OVINOCULTURA, 1., 1998, Lavras: Anais... Lavras - MG: UFLA, 2000. p. $106-128$.

BUENO, Mauro Sartori et al. Alimentação na sanidade e na reprodução ovina. In: SIMPOSIO IZ - FEINCO 2007, 2007, São Paulo: Anais... São Paulo: IZ, 2007. p.119-132.

COSTA, R.I.D. et al. Avaliação de Dois Sistema de Aeitamento com Ovinos da Raça Santa Inês no Desempenho Ponderal dos Cordeiros. In: CONGRESSO INTERNACIONAL DE ZOOTECNIA, 5., 2003. Uberaba: ABCZ. Anais... Uberaba: ABCZ: ABZ: FAZU, 2003. p. 92-95.

CUNHA, Eduardo Antônio da et al. Produção de ovinos para corte. Boletim Técnico 48, Instituto de Zootecnia: Nova Odessa, SP, 2004, 176p.

CUNHA, Eduardo Antônio da et al. Controle de Parasitas em Sistema Intensivo de Produção. In: SIMPÓSIO IZ - FEINCO 2007, 2007, São Paulo: Anais... São Paulo: IZ, 2007. p. 119-132.

FREITAS, Davi Correia de et al. Idade de Desmame de Cordeiros Deslanados para Terminação em Confinamento, no Litoral Norte da Bahia. Revista Brasileira de Zootecnia, v.34, n.4, p1392-1399, 2005. 
FRESCURA, Rafael B. Medeiros et al. Sistema de Alimentação na Produção de Cordeiros para Abate aos 28 kg. Revista Brasileira de Zootecnia, v.34, n.4, p. 1267-1277, 2005.

GARCIA, Carlos. Níveis de energia na ração de cordeiros em creepfeeding. Botucatu: Universidade Estadual Paulista, 2002. 60f. Tese (Doutorado em Zootecnia) - Universidade Estadual Paulista. 2002.

GERASEEV, Luciana Castro et al. Efeitos das Restrições Pré e Pós-Natal sobre o Crescimento e o Desempenho de Cordeiros Santa Inês do Nascimento ao Desmame. Revista Brasileira de Zootecnia, v.35, n.1, p.245-251, 2006.

GIRAO, R.N.; GIRAO, E.S.; MEDEIROS, L.P. Desenvolvimento ponderal de cordeiros da raça Santa Inês no Estado do Piauí. In: REUNIÃO ANUAL DA SOCIEDADE BRASILEIRA DE ZOOTECNIA, 36., 1999, Porto Alegre. Anais... Porto Alegre: Sociedade Brasileira e Zootecnia, 1999. p. 43-56.

PIRES, C.C.; SILVA, L.F.; SANCHEZ, L.M.B. Composição Corporal e Exigências Nutricionais de Energia e Proteína para Cordeiros em Crescimento. Revista Brasileira de Zootecnia, v.29(3), p.853-860, 2000.

PÉREZ, Juan Ramon Ololquiaga et al. Aspectos Relacionados com a Produção de Carne Ovina. In: CONGRESSO INTERNACIONAL DE ZOOTECNIA, 5., 2003. Uberaba: ABCZ. Anais... Uberaba: ABCZ:ABZ:FAZU, 2003. p. 473-489.

PÉREZ, Juan Ramon Olalquiaga et al. Aspectos Sobre o Manejo de Cordeiros. In: Ovinocultura: Aspectos Produtivos, 4, Lavras. Anais... Lavras-MG, 2005, p.59-83.

SARMENTO, José Lindenberg Rocha et al. Estatudo da Curva de Crescimento de Ovinos Santa Inês. Revista Brasileira de Zootecnia, v.35, n.2, p.435-442, 2006.

SILVA, Francisco Luiz Ribeiro; ARAÚJO, Adriana Mello. Características de Reprodução e de Crescimento de Ovinos Mestiços Santa Inês, no Ceará. Revista Brasileira de Zootecnia., v.29, n.6, p.1712-1720, 2000.

SIQUEIRA, E. R.; AMARANTE, A.F.T.; FERNANDES, S. Estudo Comparativo da Recria de Cordeiros em Confinamento e Pastagem. Veterinária e Zootecnia, v.5, p.17-28, 1993.

TONETTO, C.J. et al. Ganho de Peso e característica de carcaça de cordeiros terminados em pastagem cultivada de azevém (LolioummuliflorumLam.)e confinamento. Revista Brasileira de Zootecnia, v.33, n.1, p.225-233, 2004.

VILLAS BOAS, A.S.; Idade à desmama e manejo alimentar na produção de cordeiros superprecoces. Botucatu: Universidade Estadual Paulista, 2001. 55p. Dissertação (Mestrado em Zootecnia) - Universidade Estadual Paulista, 2001. 